This is a postprint version of the following published document:

José A. Pereda ; Ana Grande. On the Behavior of the LOD-FDTD Method at Dielectric Interfaces. In: IEEE Microwave and Wireless Components Letters, 2018, Volume 28 , Issue 6, pp. 461-463. DOI: https://doi.org/10.1109/LMWC.2018.2832014

(C) 2018 IEEE. Personal use of this material is permitted. Permission from IEEE must be obtained for all other uses, in any current or future media, including reprinting/republishing this material for advertising or promotional purposes, creating new collective works, for resale or redistribution to servers or lists, or reuse of any copyrighted component of this work in other works. 


\title{
On the behavior of the LOD-FDTD Method at Dielectric Interfaces
}

\author{
José A. Pereda and Ana Grande
}

\begin{abstract}
The electric field computed by the locally onedimensional finite-difference time-domain (LOD-FDTD) method at dielectric interfaces is investigated. To this end, two comprehensive problems are considered, namely a dielectric step in a rectangular waveguide and a dielectric-loaded metallic cavity. We found that, in both problems, the electric field patterns exhibit an unexpected phase shift at the dielectric interface. By contrast, the alternating-direction implicit (ADI)-FDTD method does not present this unphysical behavior.
\end{abstract}

Index Terms-Locally one-dimensional finite-difference timedomain (LOD-FDTD) method, alternating-direction implicit finite-difference time-domain (ADI-FDTD) method.

\section{INTRODUCTION}

Nowadays, two of the most popular unconditionally-stable finite-difference time-domain (FDTD) techniques for the numerical solution of Maxwell's equations are the alternatingdirection implicit (ADI)- and the locally one-dimensional (LOD)-FDTD methods [1]-[4].

Despite the fact that the conventional LOD-FDTD technique is first-order accurate in time only, it is usually assumed that both methods provide very similar accuracy since they exhibit the same numerical dispersion relation [2], [5]. Moreover, the comparisons reported by several researchers show that the results obtained by the LOD-FDTD method agree well with, or even duplicate, those obtained by the ADI-FDTD method. However, the range of problems considered in the literature for comparison purposes is very limited. They commonly consist of the calculation of reflection/transmission coefficients and/or resonant frequencies of empty metallic cavities. [2], [6]. The accuracy of the solution in this type of problems is essentially dependent on the numerical dispersion error. Hence, other error sources tend to remain hidden [7], [8].

In this letter we report the results obtained for two comprehensive problems comprising a dielectric interface, namely a dielectric step in a rectangular waveguide (RW) and a dielectric-loaded metallic cavity. We have focused on the study of the electric field across the dielectric-air interface. We have found an important flaw in the LOD-FDTD method which has not been reported to date. In both problems, the electric field patterns computed by the LOD-FDTD method exhibit an unexpected phase shift at the dielectric interface. Moreover,

Manuscript received December 20, 2017. This work was supported in part by the Spanish Government (MINECO) and the European Commission (ERDF) under Research Projects TEC2014-55463-C3-2-P and TEC201455463-C3-3-P

J. A. Pereda is with the Dpto. de Ingeniería de Comunicaciones (DICOM), Universidad de Cantabria, Avda. Los Castros s/n, 39005 Santander, Cantabria, Spain (email: peredaj@unican.es).

A. Grande is with the Dpto. de Electricidad y Electrónica, Universidad de Valladolid, 47011 Valladolid, Spain (email: agrande@uva.es). a jump in magnitude is also observed for large values of the time step. By contrast, the electric field patterns computed, under the same conditions, by the ADI-FDTD method do not present this unphysical behavior.

\section{THEORETICAL BACKGROUND}

Consider $\mathrm{TM}^{z}$ waves in linear, isotropic, nondispersive, lossless media with permittivity $\epsilon$ and permeability $\mu$. The conventional LOD-FDTD method comprises two steps in each time iteration, which can be expressed in matrix form as [2]

$$
\begin{aligned}
& \left(\mathbf{I}-\frac{\Delta_{t}}{2} \mathbf{A}\right) \vec{U}^{n+\frac{1}{2}}=\left(\mathbf{I}+\frac{\Delta_{t}}{2} \mathbf{A}\right) \vec{U}^{n} \\
& \left(\mathbf{I}-\frac{\Delta_{t}}{2} \mathbf{B}\right) \vec{U}^{n+1}=\left(\mathbf{I}+\frac{\Delta_{t}}{2} \mathbf{B}\right) \vec{U}^{n+\frac{1}{2}}
\end{aligned}
$$

where $\Delta_{t}$ is the time step, $\mathbf{I}$ is the identity matrix, $\vec{U}=$ $\left[E_{z}, H_{x}, H_{y}\right]^{\mathrm{T}}$ and

$$
\mathbf{A}=\left[\begin{array}{ccc}
0 & 0 & \frac{\delta_{x}}{\epsilon \Delta_{x}} \\
0 & 0 & 0 \\
\frac{\delta_{x}}{\mu \Delta_{x}} & 0 & 0
\end{array}\right], \mathbf{B}=\left[\begin{array}{ccc}
0 & -\frac{\delta_{y}}{\epsilon \Delta_{y}} & 0 \\
-\frac{\delta_{y}}{\mu \Delta_{y}} & 0 & 0 \\
0 & 0 & 0
\end{array}\right]
$$

where $\delta_{x}$ and $\delta_{y}$ are second-order central-difference operators and $\Delta_{x} \times \Delta_{y}$ the size of the spatial unit cell

The maximum time step allowed in the original Yee-FDTD method, $\Delta_{t, \max }$, is limited by the Courant stability condition [9]. However, for unconditionally-stable schemes, such as the LOD- and the ADI-FDTD methods, $\Delta_{t}$ is only limited by accuracy considerations. When using these methods, instead of directly giving the time step used in a simulation, one can provide the stability factor defined as $s=\Delta_{t} / \Delta_{t, \max }$. It is also interesting to provide the temporal resolution associated with the time step used, which is defined as $N_{t}=T / \Delta_{t}$, where $T$ is the period of the wave.

\section{NUMERICAL EXAMPLES}

As a first example, we consider the discontinuity problem between a dielectric-filled and an empty RWs, as shown in the inset of Fig. 3. The waveguide width is $a=19.05$ $\mathrm{mm}$ and the relative permittivity $\epsilon_{r}=16$. We assume the incidence of a $\mathrm{TE}_{10}^{y}$ mode towards $y>0$ at the frequency $f=0.4 f_{c} \simeq 3.15 \mathrm{GHz}$, where $f_{c}$ is the cutoff frequency of the empty waveguide. Since the empty RW is below cutoff, total reflection takes place at the dielectric interface $(y=0)$. Hence, a standing wave is formed in the dielectric region while fields are evanescent (i.e. they decay exponentially) in the empty region. The penetration depth in the empty RW is $d=1 / \alpha \simeq 6.62 \mathrm{~mm}$, with $\alpha$ being the attenuation constant. 


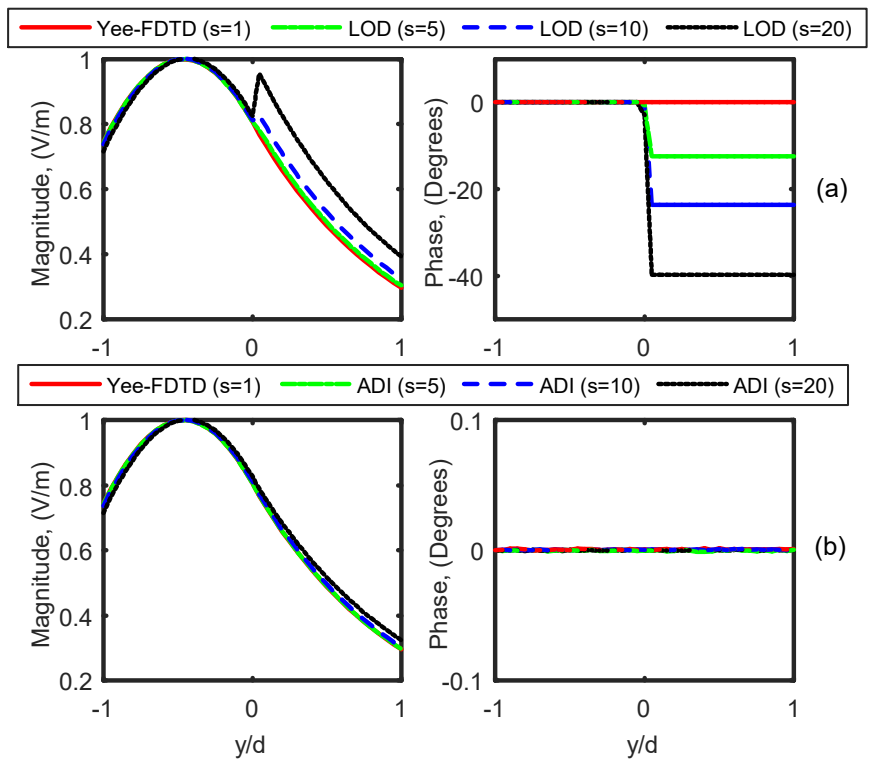

Fig. 1. Electric field pattern calculated by (a) the LOD- and (b) the ADIFDTD methods at $x=a / 2$ for the waveguide discontinuity shown in the inset of Fig. 3. The Yee-FDTD results are also included. (a)

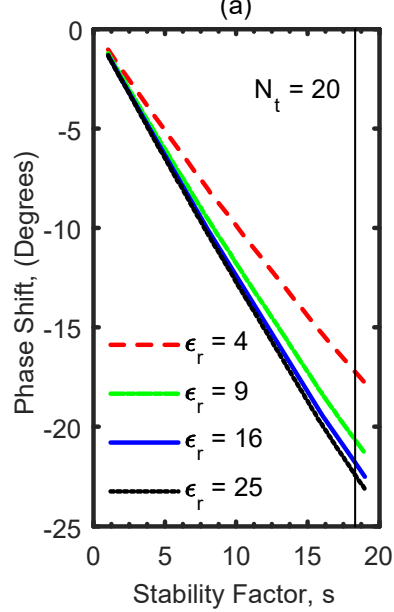

(b)

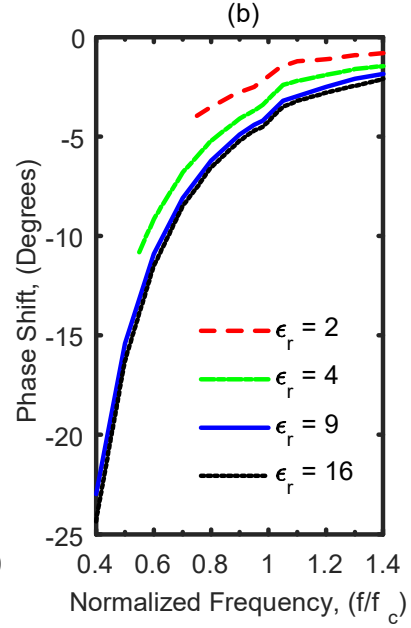

Fig. 2. (a) Phase shift against the stability factor for $f=0.6 f_{c}$. (b) Phase shift against the frequency for a temporal resolution $N_{t}=40$.

The size of the spatial cell was $\Delta_{x}=a / 60 \simeq 0.32 \mathrm{~mm}$ and $\Delta_{y}=d / 20 \simeq 0.33 \mathrm{~mm}$. Both RW ports have been terminated by second-order absorbing boundary conditions located far enough from the discontinuity [10].

Fig. 1a shows the magnitude and phase of the electric field pattern at $x=a / 2$ computed by the LOD-FDTD method for $s=5,10$ and 20 . The results obtained by the Yee-FDTD method with $s=1$ are also included as a reference. It can be seen that the LOD-FDTD solutions exhibit an unexpected phase shift at the dielectric interface. Moreover, a magnitude jump is also evident for $s=20\left(N_{t} \simeq 20.8\right)$. For comparison purposes, Fig. $1 \mathrm{~b}$ depicts the results obtained by the ADIFDTD method under the same conditions as those in Fig. 1a. Note that the field patterns calculated by the ADI-FDTD

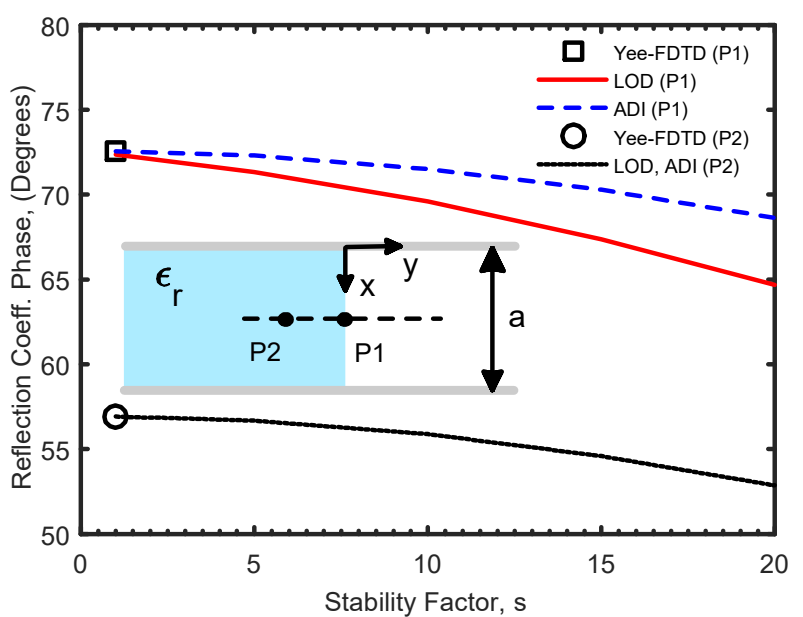

Fig. 3. Reflection coefficient phase calculated at points P1 and P2 shown in the inset (top view of the RW).

method do not present any anomalous jump.

To further investigate the nature of the abnormal field discontinuity found when using the LOD-FDTD method, Fig. 2a shows the phase shift against $s$ for $f=0.6 f_{c} \simeq 4.72 \mathrm{GHz}$ and for several values of $\epsilon_{r}$. It can be seen that there is phase shift even for small values of $s$. Moreover, this shift increases with $\epsilon_{r}$ and, approximately linearly, with $s$ (i.e. with $\Delta_{t}$ ). In Fig. $2 \mathrm{~b}$ the phase shift is represented as a function of $f / f_{c}$ for $N_{t}=40$ and for several values of $\epsilon_{r}$. Note that the phase shift decreases as the frequency increases, being less significant when both RWs are above cutoff $\left(f / f_{c}>1\right)$. All the results in Fig. 2 have been calculated with $\Delta_{x}=a / 80$ and with $\Delta_{y}=d / 30$ for $f / f_{c}<1$ or $\Delta_{y}=\lambda_{g d} / 50$ for $f / f_{c}>1$, where $\lambda_{g d}$ is the guided wavelength in the dielectric-filled RW. Anyway, according to our experience, the phase shift under study does not vary with the size of the spatial cell.

Fig. 3 shows the reflection coefficient phase as a function of $s$ calculated by the LOD-, ADI- and Yee-FDTD methods at points $\mathrm{P} 1$ and $\mathrm{P} 2$ (shown in the inset). The calculation at $\mathrm{P} 1$ is carried out by recording the electric field directly at the dielectric interface. For this case, the LOD- and the ADIFDTD methods provide different results. More specifically, the accuracy decreases faster with $s$ for the LOD than for the ADI case. However, at P2, which is two cells inside the dielectric region, the two methods provide exactly the same results.

As a second example, we consider a rectangular metallic cavity partially filled with a dielectric of $\epsilon_{r}=16$, as shown in the inset of Fig. 4. The size of the cavity is $a=10 \mathrm{~mm}$ and $b=1.5 a$. The resonant frequency of the dominant mode was computed by using $60 \times 60$ spatial cells. This frequency was obtained from the first amplitude peak of the discrete Fourier transform of the electric field recorded at a selected point of the cavity. The same set-up was used for the three FDTD methods. The Yee-FDTD solution with $s=1$ is $f_{1,1}=5.6047$ GHz. Taking this result as reference, Fig. 4 shows the resonant frequency relative error against $s$. It can be seen that both the LOD- and the ADI-FDTD methods provide the same results.

To assess the accuracy of the fields provided by each 


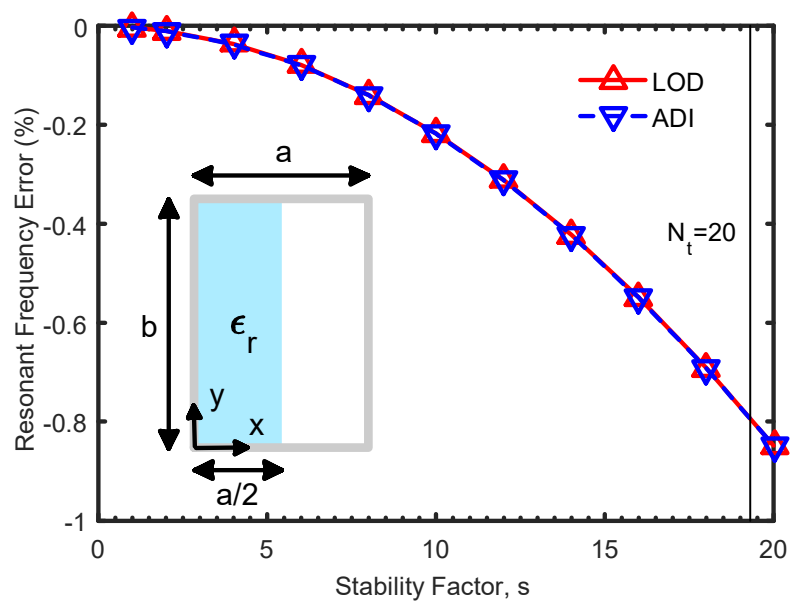

Fig. 4. Resonant frequency relative error against the stability factor for the dominant mode of the dielectric-loaded cavity shown in the inset.

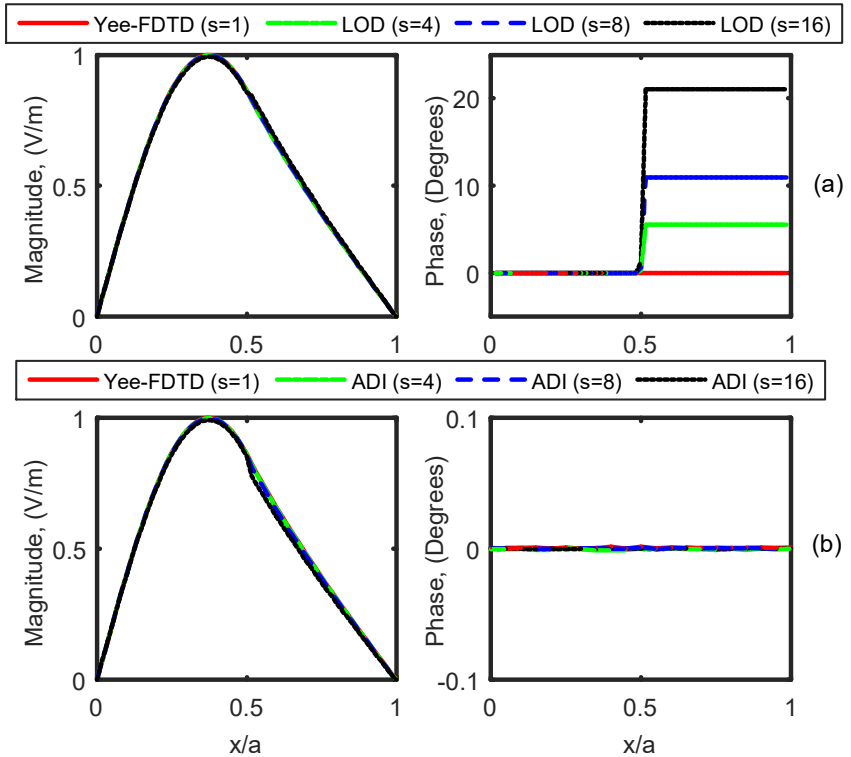

Fig. 5. Electric field pattern calculated by (a) the LOD- and (b) the ADIFDTD methods at $y=b / 2$ for the dominant mode of the dielectric-loaded cavity shown in the inset of Fig. 4 . The Yee-FDTD results are also included.

method, Fig. 5 shows the electric field pattern along the $x$ direction for $y=b / 2$ for the dominant mode of the cavity. The phase obtained by the LOD-FDTD method again exhibits an unexpected shift at the dielectric interface. On the contrary, the ADI-FDTD technique does not present any field jump.

Finally, Fig. 6 plots a snapshot of the electric field calculated at the time iteration 12495 with $s=16\left(N_{t} \simeq 24\right)$ for the dominant mode of the cavity. Note that, for the LOD-FDTD method, the electric field in the dielectric is positive (red) while it is negative (blue) in the air. However, for the ADI case, it is positive in the whole resonator, as expected.

\section{CONCLUSION}

Contrary to what happens in the ADI case, the electric field patterns computed by the conventional LOD-FDTD method

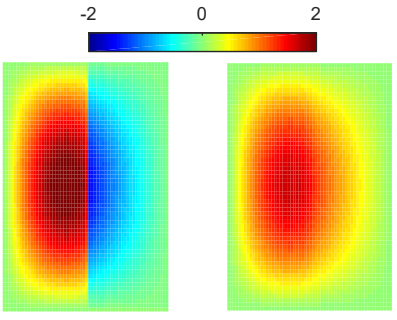

(a)

(b)

Fig. 6. Electric field snapshot at the time iteration 12495 calculated with $s=16$ for the dominant mode of the resonant cavity shown in the inset of Fig. 4. (a) LOD-FDTD, (b) ADI-FDTD

exhibit anomalous phase shift at dielectric interfaces. The extent of the phase shift increases primarily with $\Delta_{t}$ and it is very significant when evanescent fields are involved. This phenomenon can be ascribed to the fact that the local truncation error of the LOD-FDTD method exhibits first-order error terms that depend on $\Delta_{t}$ and the spatial derivatives of the fields. These terms are not present in the ADI scheme [8]. Consequently, the conventional LOD-FDTD method should be avoided when one is concerned with the accurate computation of near-fields and/or the interpretation of physical phenomena involving them.

Despite this pitfall, the resonant frequencies and scattering parameters (if properly computed) provided by the LODFDTD method are the same as those obtained by the ADIFDTD technique. This behavior is consistent with the fact that both methods exhibit the same numerical dispersion relation.

In the light of the above results, the study of the accuracy of second-order LOD-FDTD based methods, such as the one introduced in [3], would be of great interest.

\section{REFERENCES}

[1] T. Namiki, "3-D ADI-FDTD method: unconditionally stable time domain algorithm for solving full vector Maxwell's equations," IEEE Trans. Microw. Theory Tech., vol. 48, no. 10, pp. 1743-1748, Oct. 2000.

[2] J. Shibayama, M. Muraki, J. Yamauchi, and H. Nakano, "Efficient implicit FDTD algorithm based on locally one-dimensional scheme,' Electron. Lett., vol. 41, no. 19, pp. 1046-1047, Sep. 2005.

[3] E. L. Tan, "Unconditionally stable LOD-FDTD method for 3-D Maxwell's equations," IEEE Microw. Wireless Compon. Lett., vol. 17, no. 2, pp. 85-87, Feb. 2007.

[4] E. L. Tan, "Fundamental schemes for efficient unconditionally stable implicit finite-difference time-domain methods", IEEE Trans. Antennas Propag., vol. 56, no. 1, pp. 170-177, Jan. 2008.

[5] Q. F. Liu, Z. Chen, and W. Y. Yin, "An arbitrary-order LOD-FDTD method and its stability and numerical dispersion," IEEE Trans. Antennas Propag., vol. 57, no. 8, pp. 2409-2417, Aug. 2009.

[6] A. k. Saxena and K. V. Srivastava, "A three-dimensional unconditionally stable five-step LOD-FDTD method," IEEE Trans. Antennas Propag., vol. 62, no. 3, pp. 1321-1329, Mar. 2014

[7] S. G. García, T.-W. Lee, and S. C. Hagness, "On the accuracy of the ADI-FDTD method," IEEE Antennas Wireless Propag. Lett., vol. 1, pp. $31-34,2002$

[8] A. Grande and J. A. Pereda, "Accuracy limitations of the locally onedimensional FDTD technique," IEEE Microw. Wireless Compon. Lett., vol. 13, pp. 1180-1183, Jun. 2014.

[9] A. Taflove and S. C. Hagness, Computational Electrodynamics-The Finite-Difference Time-Domain Method, 3rd ed. Norwood, MA: Artech House, 2005.

[10] J. A. Pereda, A. Serroukh, A. Grande, and A. Vegas, "Implementation of absorbing boundary conditions based on the second-order one-way wave equation in the LOD- and the ADI-FDTD methods," IEEE Antennas Wireless Propag. Lett., vol. 11, pp. 981-983, 2012. 